\title{
Maternal Death following Induction of Labour: A Case Report with Uncommon Findings of Amniotic Fluid Embolism
}

\author{
Jayarathne $\mathrm{UGB}^{1}$, Warushahennadi $\mathrm{J}^{2 *}$, Perera $\mathrm{C}^{2}$ \\ ${ }^{1}$ Teaching Hospital, Karapitiya, Sri Lanka, ${ }^{2}$ Department of Forensic Medicine, Faculty of Medicine, University \\ of Ruhuna, Sri Lanka.
}

\begin{abstract}
Amniotic fluid embolism (AFE) is a rare but potentially catastrophic obstetric emergency characterized by a combination of signs and symptoms that reflect sudden cardiorespiratory collapse and disseminated intravascular coagulation (DIC). The diagnosis of AFE has traditionally been made at autopsy when the amniotic debris is found in the maternal pulmonary vasculature. A 28 years old term mother was admitted to the hospital for confinement. One hour after the induction of labour she complained of difficulty in breathing and excessive sweating and in spite of the intensive resuscitation measures she was pronounced dead. The autopsy examination was unremarkable except for the diffuse extensive retroperitoneal haemorrhage. The histology sections from lungs show amniotic fluid components; mainly cuticulated epithelium, foetal squamous cells and keratinized epithelial cells in small arteries and capillaries. Similar findings were observed in the histology sections obtained from the liver, kidney, brain, myometrium, and myocardium. The value of histopathological confirmation using special stains is emphasized in this case report.
\end{abstract}

Keywords: Amniotic fluid embolism, histopathology, amniotic fluid debris

Received: 25 Nov 2020, Revised version accepted: 29 Dec 2020, Published: 31 Dec 2020. *Corresponding author: Warushahennadi J, $\triangle$ Email: janakiwh@gmail.com (D) https://orcid.org/0000-0002-6880-5513

Cite this article as: Jayarathne UGB, Warushahennadi J, Perera C. Maternal Death following Induction of Labour - A Case Report with Uncommon Findings of Amniotic Fluid Embolism. Medico-Legal Journal of Sri Lanka, 2020;8(2):36-39. DOI: http://dx.doi.org/10.4038/mljsl.v8i2.7424

Copyright: @ 2019 with the Medico-legal Journal of Sri Lanka.

This is an open-access article distributed under the terms of the Creative Commons Attribution 4.0 International License, which permits unrestricted use, distribution, and reproduction in any medium provided the original author and source are credited.

\section{Introduction}

Amniotic fluid embolism (AFE) is a rare but potentially catastrophic obstetric emergency characterized by a combination of signs and symptoms that reflect sudden cardiorespiratory collapse and disseminated intravascular coagulation (DIC). ${ }^{[1]}$ Despite the early detection and prompt treatment, morbidity and mortality rates remain high. The diagnosis of AFE has traditionally been made at autopsy when the amniotic debris is found in the maternal pulmonary vasculature. ${ }^{[2]}$

Although the pathophysiology of AFE is not completely understood, the process is more similar to anaphylaxis than to embolism, so the term anaphylactoid syndrome of pregnancy has been suggested because amniotic fluid components are not universally found in mothers who present with signs and symptoms of AFE. ${ }^{[3]} \mathrm{We}$ report an uncommon presentation of widespread AFE which was confirmed by histo-pathologically using different special stains.

\section{Case report}

A 28-year-old term mother (P2, C1) with unremarkable antenatal history was admitted to a tertiary care hospital for confinement. The induction of the labour was started with artificial rupture of the membranes (ARM) and with an infusion of oxytocin.

One hour later she complained of difficulty in breathing and excessive sweating. The examination revealed hypotension, tachycardia, and foetal monitoring by cardiotocograph (CTG) showed foetal distress with bradycardia. Shortly she became unresponsive and with the Cardio Pulmonary Resuscitation an emergency uneventful Caesarean section was performed and a live baby was delivered. In spite of the intensive resuscitation measures, she was pronounced dead.

The medico-legal autopsy was performed in the presence of the Obstetric and the Anaesthetic teams. The external examination revealed evidence of recent pregnancy and medical and surgical interventions. Sutured Caesarean section incision and an abdominal drain were observed. Internal examination revealed $200 \mathrm{ml}$ of blood-stained pleural fluid in both pleural cavities and approximately $20 \mathrm{ml}$ of blood-stained pericardial fluid. There were numerous petechial haemorrhages 
over the visceral surfaces of the lungs and pericardium. Both lungs were congested with moderate pulmonary oedema. A diffuse extensive retroperitoneal bleeding was observed.(Figure 01) The internal organs including the brain were pale.

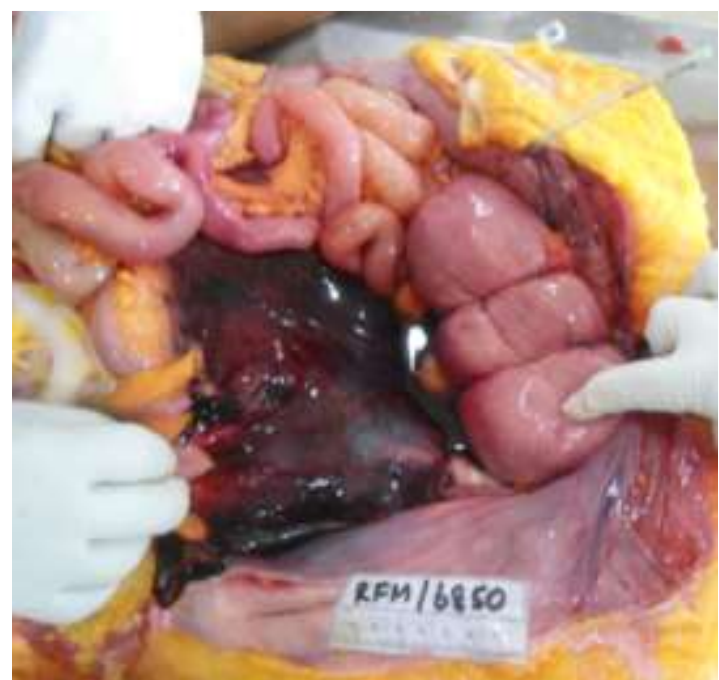

Figure 01. Abdominal cavity is opened. Note retroperitoneal haemorrhage

Tissue samples from the internal organs including multiple tissue samples from each lobe of the lungs were obtained for histology. The tissues were initially stained with $\mathrm{H} \& \mathrm{E}$ and detected amniotic fluid components; mainly cuticulated epithelium, foetal squamous cells and keratinized epithelial cells in small arteries and capillaries in the sections from lungs, brain, kidneys, liver, spleen, heart and myometrium. (Figure 2-6)

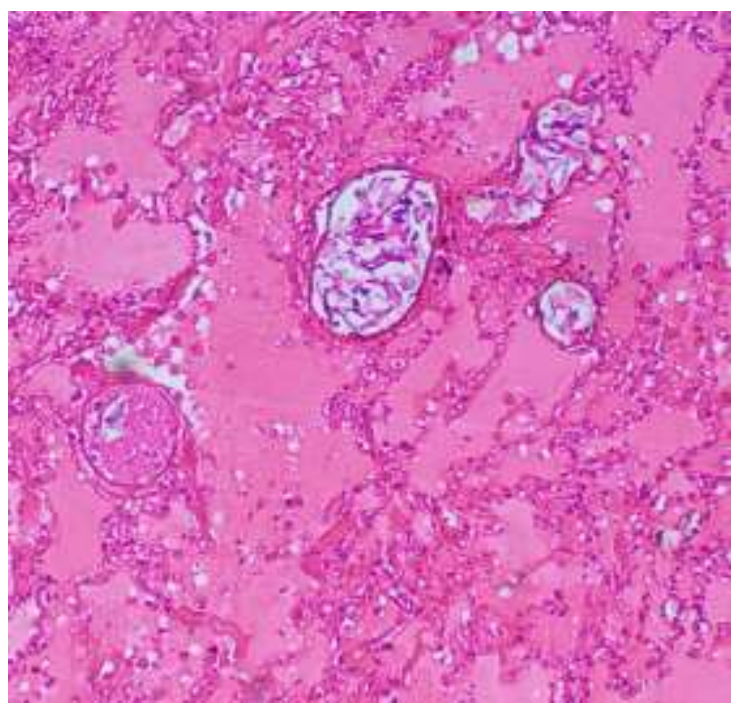

Figure 02. Photomicrograph of lungs

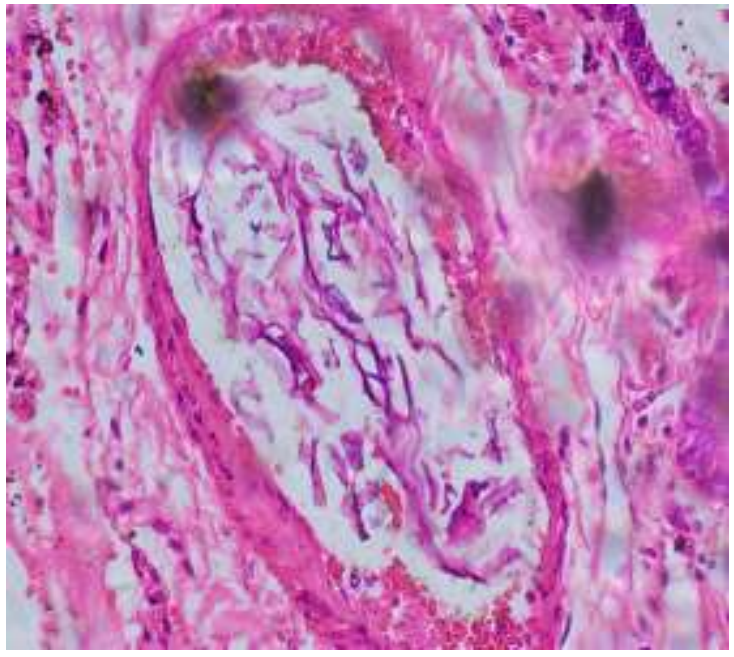

Figure 03. Photomicrograph of lungs

Figure 02 and 03 shows Histopathology of the lungs. Note the amniotic fluid components in the small arteries, oedema fluid in alveoli and interstitium.

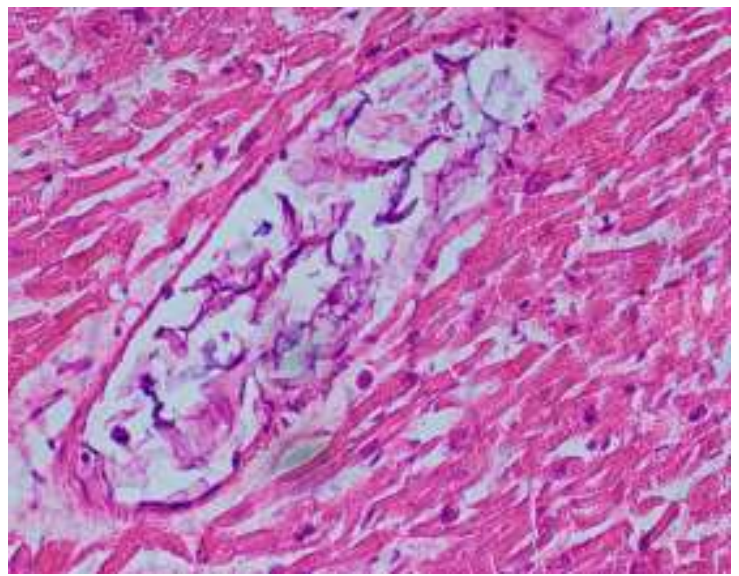

Figure 04: Photomicrograph of the myocardium

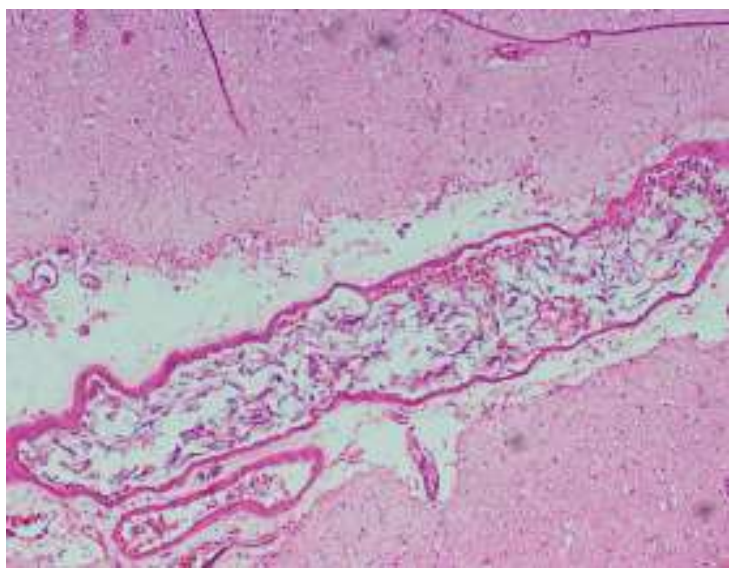

Figure 05: Photomicrograph of brain 


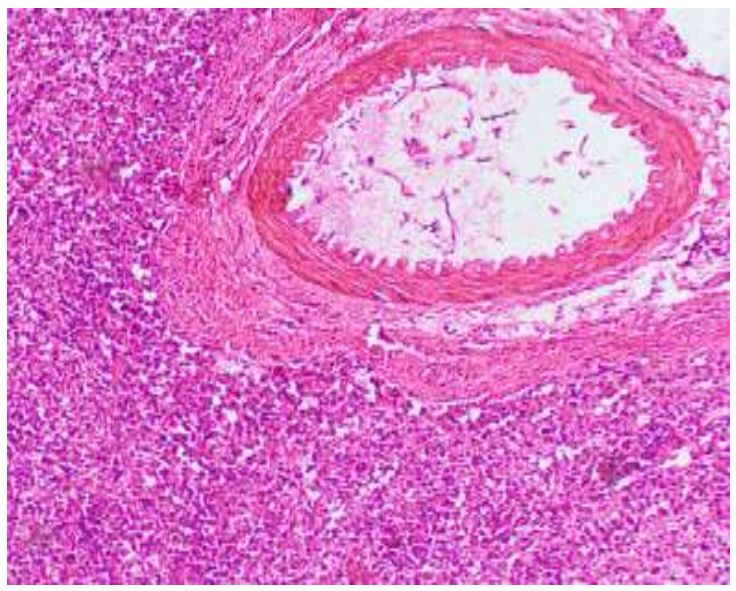

Figure 06. Photomicrograph of spleen

The lung histology showed dilated as well as collapsed alveolar spaces, marked intra-alveolar septal oedema, congested blood vessels and foci of pulmonary haemorrhages. A Transparent fibrin microthrombi was observed in some lung capillaries.

Sections of several lung tissues were stained with Alcian Blue and detected mucin particles of epithelial cells of amneotic fluid and further the immunohistochemistry with cytokeratin 5,6 (CK56) detected marked epithelial squamous in the pulmonary vasculature. (Figure 7, 8) Considering the typical histopathology the cause of death was concluded as AFE.

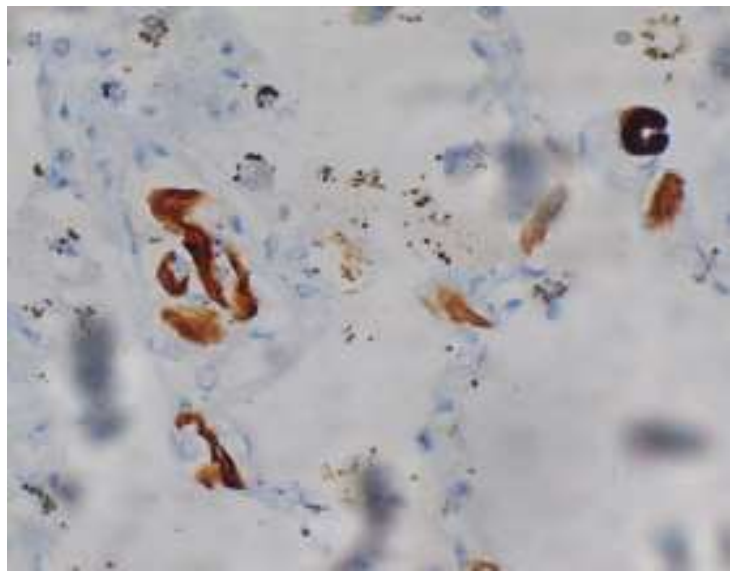

Figure 07. Histopathology Immunohistochemistry - CK56. Note keratin

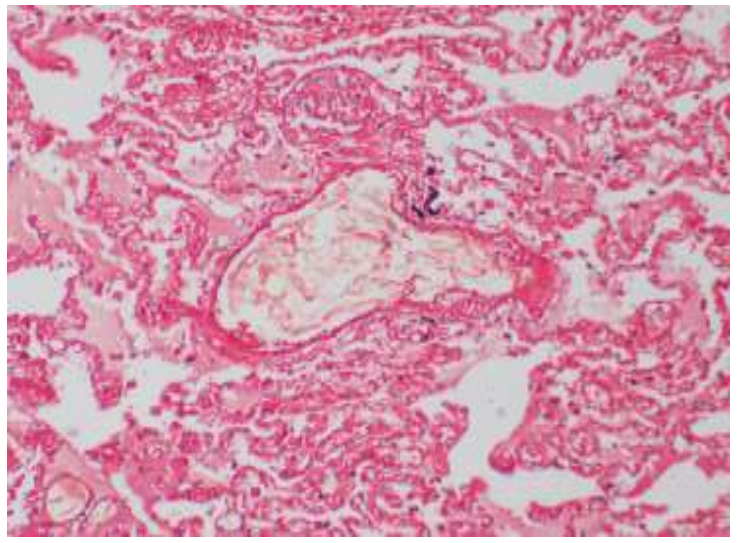

Fig. 08. Photomicrograph of lung tissue with Alcian Blue stain

\section{Discussion}

AFE is a rare obstetric complication which typically occurs during labour and delivery or in the immediate postpartum period. The patients with a rapid onset of AFE may die within a few minutes or after recovery often suffer post-partum haemorrhage, DIC and finally multiple organ failure followed by renal, respiratory and cardiac failure. ${ }^{[4]}$

The determination of the cause of death in these obscure autopsies is a challenging task for the forensic pathologist. The clinical presentation in combination with a lack of other explanations for the maternal cardiovascular collapse and postmortem findings with ancillary investigations support to determine the cause of death. The patient presented with typical clinical signs and symptoms of AFE which were able to elicit from the clinical records and the discussions with clinicians during the autopsy.

The confirmation of the AFE is mainly dependent on the histology findings. But the mere presence of foetal squamous cells in the pulmonary circulation is not necessarily diagnostic for AFE, as some squamous cells have been found in the pulmonary circulation of pregnant women without the diagnosis of AFE. ${ }^{[5]}$

The presence of amniotic fluid debris in this case was confirmed with Alcian blue stains and immunohistochemistry. The unusual finding of this case was that the amniotic fluid debris was detected in H\&E stains in other organs particularly the brain, heart, kidney, liver, myometrium and spleen.

The dilated as well as collapsed alveolar spaces, marked intra alveolar septal oedema, congested blood vessels and foci of pulmonary haemorrhages were consistent with acute lung injury. The retroperitoneal haemorrhage and the petechial haemorrhages may be due to the effects of DIC. 


\section{Conclusions}

The value of evaluation of the history, thorough external and internal autopsy examination and histopathological confirmation using special stains are emphasized in this report.

\section{Acknowledgments}

We wish to acknowledge Dr. Padmini Kariyawasam, Consultant Histopathologist, Teaching Hospital, Karapitiya for arranging histopathology special staining and Dr. MKL Manjula, Consultant Histopathologist, Base Hospital, Hambantota for the guidance given in the interpretation of the histopathology slides.

\section{Disclosure statement}

Conflicts of interest: The authors declare that they have no conflicts of interest.

Funding: None

\section{References}

1 Ito F, Akasaka J, Koike N, Uekuri C, Shigemitsu A, Kobayashi H. Incidence, diagnosis and pathophysiology of amniotic fluid embolism. J Obstet Gynaecol 2014; 34(7): 580-4

2 Fitzpatrick KE, Tuffnell D, Kurinczuk JJ, Knight M. Incidence, risk factors, management and outcomes of amniotic-fluid embolism: a population-based cohort and nested case-control study. BJOG 2016; 123(1):100-9

3 Hikiji W, Tamura N, Shigeta A, Kanayama N, Fukunaga T. Fatal amniotic fluid embolism with typical pathohistological, histochemical and clinical features. Forensic Sci Int 2013; 226 $(1-3): 16-19$.

4 Stehr SN, Liebich I, Kamin G, Koch T, Litz RJ: Closing the gap between decision and deliveryamniotic fluid embolism with severe cardiopulmonary and haemostatic complications with a good outcome. Resuscitation 2007;74 (2):377-81

5 Benson MD, Kobayashi H, Silver RK, Oi H, Greenberger PA, Terao T: Immunologic studies in presumed amniotic fluid embolism. Obstet Gynecol 2001;97(4):510-4. 